\title{
Differentiation of PEDV Classical Attenuated Vaccine Strains from Wild-type Strains using One- Step Real-Time Fluorescent Reverse Transcription PCR Assay Targeting ORF1 Nucleotides Deletion Region
}

\section{Zhilin Wang}

Lanzhou Veterinary Research Institute

Xuerui Li

Lanzhou Veterinary Research Institute

Youjun Shang

Lanzhou Veterinary Research Institute

Jinyan Wu

Lanzhou Veterinary Research Institute

Zhen Dong

Lanzhou Veterinary Research Institute

Wanning Wang

Lanzhou Veterinary Research Institute

Yongsheng Liu

Lanzhou Veterinary Research Institute

Xi Lan ( $\sim$ lanxi@caas.cn )

Lanzhou Veterinary Research Institute https://orcid.org/0000-0001-7073-8818

\section{Research}

Keywords: PEDV, real-time RT-PCR, differentiate, ORF1

Posted Date: May 29th, 2021

DOI: https://doi.org/10.21203/rs.3.rs-529609/v1

License: (c) (i) This work is licensed under a Creative Commons Attribution 4.0 International License.

Read Full License 


\section{Abstract}

\section{Background}

Porcine epidemic diarrhea virus (PEDV) is a pathogen causing serious disease and resulting in severe economic losses in the swine industry. In recent years, although China has adopted a large-scale vaccine immunization strategy, many types of PEDV strains, including classical attenuated vaccine strains, have been discovered in the immunized pig herds. Therefore, monitoring the prevalence of different types of PEDV strains is particularly important for the production of pigs and the safety evaluation of related attenuated vaccines

Methods

In the study, a one-step real-time fluorescent reverse transcription PCR (one-step real-time RT-PCR) assay targeting 24-nucleotide deletion in the ORF1 region of three PEDV classical attenuated vaccine strains (derived from classical strains) was established, which could effectively distinguish PEDV classical attenuated vaccine strains and wild-type strains.

Results

In our study, the RNA detection limits for PEDV wild-type strains and classical attenuated vaccine strains were $3.0 \times 10^{3}$ copies and $3.0 \times 10^{2}$ copies, respectively. This assay was highly specific for PEDV, with no cross-reactivity for other viruses, causing diarrheal disease. A total of 117 swine fecal samples were analysed by this established real-time RT-PCR assay, indicating that classical attenuated vaccine strains were present in the swine herds in Gansu province, China. Additionally, a pair of primers and two probes of the established assay can be placed in one reaction tube to distinguish PEDV classical attenuated vaccine strains and wild-type strains.

Conclusion

Our results provided an effective and cheap technology platform for clinical rapid identification testing and epidemiological investigations of PEDV wild-type strains and classical attenuated vaccine strains

\section{Background}

Porcine epidemic diarrhea virus (PEDV), an enveloped virus belonging to the genus Alpha coronavirus and the family Coronaviridae, can induce acute diarrhea and vomiting in newborn suckling and weaning pigs[1]. In 1971, PED was first reported among feeder and fattening pigs in England[2]. Since then, the diarrheal disease caused by PEDV has broken out in many regions of the world, including China[3-5]. After 2010, the highly pathogenic PEDV variant strains cause the morbidity rate of newborn suckling piglets to reach $100 \%$, with mortality rates of $80-100 \%$, which result in significant economic losses in the global swine industry[6-8]. Since the 1990s, many Chinese pig farms have adopted vaccination measures to prevent the spread of the disease[9]. At present, CV777-based live attenuated vaccine and 
PEDV(CV777) and transmissible gastroenteritis virus (TGEV)-based dual attenuated vaccines have been extensively applied for PED prevention in China[10,11]. There is a short period of carrying attenuated vaccine virus in the orally immunized pig population, and the possibility that the wild-type strains and the attenuated vaccine strains will exist simultaneously in the pig herds[12, 13]. In recent study, multiple strains were also found in many immunized pig population in China $[4,14,15]$, and phylogenetic analysis of the whole genome also showed that there are multiple variation sites in PEDV genome[16, 17]. The antigenicity of vaccines derived from classical vaccine strains may be altered due to these genetic mutations, resulting in low vaccination efficiency and inability to protect pigs from variant PEDV strains[16]. Those have an impact on accuracy of the epidemiological survey data of the disease to a certain extent.

Porcine epidemic diarrhea virus (PEDV) is a positive-sense, single-stranded virus RNA with a genome of about $28 \mathrm{~kb}$ in length[1]. The PEDV genome comprises seven open reading frames in the order of ORF1a, ORF1b, spike (S) gene, accessory gene ORF3, envelope (E) gene, membrane (M) gene and nucleocapsid $(\mathrm{N})$ gene[3, 18, 19]. The ORF1a and ORF1b region at the 5 'end of the genome occupy $2 / 3$ of the entire genome, and the two viral replicase polyproteins (pp1a and pp1ab) encoded by them are cleaved into 16 non-structural proteins (nsp1-nsp16), which are involved in the synthesis and transcription of viral RNA[20]. Among them, the nsp3 is the largest non-structural protein and also a multifunctional protein, which may be closely related to virus replication and transcription[21, 22]. A recent variation analysis of 49 PEDV entire-genome sequences in GenBank showed that the N-terminal domain of PEDV nsp3 is a hypervariable region and relatively conservative, and the characteristic mutation sites in this region may potentially serve as markers for the classification of PEDV different genotypes[16, 23]. Currently, many molecular detection methods targeting N, M, S, ORF3 genes have been developed in the laboratory[24-27], such as traditional PCR[13, 28], nanoparticle-assisted RT-PCR[29], and real-time RT-PCR[12, 27, 30] methods to detect or distinguish the wild-type strains (classical wild strains and variant strains) and attenuated vaccine strains, these methods is useful for understanding the epidemic status of different strains. In previous studies, researchers rarely used the ORF1 region as the genetic characteristics to distinguish PEDV attenuated vaccine strains and wild-type strains. In present study, we found that Nterminal domain of ORF1 region nsp3 in the three PEDV classical attenuated vaccine strains (PEDV attenuated vaccine strain KC189944, attenuated CV777 and DR13) and five Vero-cell-adapted isolates (JS2008, SDM, SQ2014, SC1402, HLJBY) bore a 24-nucleotide deletion (see Fig.1 and Table S1) compared with 38 PEDV wild-type strains whose sequences were available in GenBank[31]. These three attenuated vaccine strains and five Vero cell-adapted isolates were artificially cell-passaged and were not original strains in nature. Unless they are used as live attenuated vaccines for vaccination and spread to the pig population, it is impossible to detect exactly the same strains in the field. Due to these discoveries, we developed a convenient, cheap, efficient, secure and reliable one-step real-time RT-PCR assay to distinguish PEDV classical attenuated vaccine strains and wild-type strains.

\section{Methods}

\section{Viruses and clinical samples}


PEDV attenuated vaccine strain CV777 and Vero-cell-adapted isolate JS2008 were passaged in Vero E6 cell. Nucleic acid products and clinical samples of PEDV wild-type strain DX, transmissible gastroenteritis virus (TGEV), porcine circovirus type 2(PCV-2), Porcine deltacoronavirus(PDCoV), Porcine parvovirus (PPV), and porcine kobuvirus (PKV) were preserved in our laboratory. All 117 swine fecal samples with suspected PEDV infection were obtained from seven swine farms in Lanzhou, Dingxi, Baiyin, Jiayuguan, Linxia and Tianshui, Gansu province, China, between October 2015 and June 2018. All samples were stored at $-80^{\circ} \mathrm{C}$ until use.

\section{DNA/RNA extraction}

All clinical samples were centrifuged at $4000 \mathrm{~g}$ for $15 \mathrm{~min}$ and the supernatants were stored at $-80^{\circ} \mathrm{C}$.Viral RNA and DNA were extracted using the TaKaRa MiniBEST Viral RNA/DNA Extraction Kit Version 5.0 (Takara, Dalian, China) according to the manufacturer's instructions. Viral RNA or DNA of each sample was eluted in $30 \mu \mathrm{L}$ of RNase-free water. All RNA and DNA samples were stored at $-80^{\circ} \mathrm{C}$ until use.

\section{Design of primers and probes}

According to the presence of a 24-nucleotide deletion (Fig.1) in the ORF1 regions of three PEDV classical attenuated vaccine strains and five Vero-cell-adapted isolates, a pair of primers and two probes were designed (synthesized by Sangon Biotech, Shanghai, China) to differentiate PEDV classical attenuated vaccine strains from wild-type strains (Table 1). Additional primers and probes(Table S2) (synthesized by Sangon Biotech, Shanghai, China) were synthesized to detect PEDV, TGEV, PKV, PPV, PDCoV, and PCV-2 in the Fecal samples.

\section{Table1. Primers and probes used in the established real time RT-PCR assay}

\begin{tabular}{|c|c|c|c|}
\hline Name & Primers or probes Sequence $\left(5^{\prime}-3^{\prime}\right)$ & Location & Length (bp) \\
\hline F3365 & GGTGGCAGATGTGGCTAACT & ORF1,3365-3384 ${ }^{\mathrm{a}}$ & \\
\hline R3445 & AATAAAGGACAAAGTTGCGGC & ORF1,3445-3425 & $185 b p$ \\
\hline $\begin{array}{l}\text { P3390- } \\
\text { W }\end{array}$ & FAM-AGGATGATGGTCTTAATGTAGCTCCTGAA-BHQ1 & ORF1,3390-3418 & \\
\hline P3388-V & ROX-TGAGGCTGATGATGTAGAGTCTGAAGT-BHQ2 & ORF1,3388-3414 ${ }^{\mathrm{a}}$ & \\
\hline F-V & CACCGATCCTAATCTGCCCG & ORF1,3217-3236 ${ }^{\mathrm{a}}$ & \\
\hline $\mathrm{R}-\mathrm{V}$ & TGGACCAACTCTACCAGCAC & ORF1,3612-3632 & $415 b p$ \\
\hline F-W & ACACTATACTATCCCACCG & ORF1,2915-2932 & \\
\hline$R-W$ & CACCCAAAGATCCCAAGA & ORF1,3690-3708b & 793bp \\
\hline
\end{tabular}

379. PEDV classical attenuated vaccine strain CV777, GenBank accession number KT323979.1 
380. PEDV wild-type strain AJ1102, GenBank accession number JX188454.1

\section{Generation of RNA standards}

The first strand PEDV classical attenuated vaccine strain CV777 and wild strain DX cDNA were synthesized by reverse transcription with PrimeScript ${ }^{\mathrm{TM}}$ first strand cDNA synthesis kit (Takara, Dalian, China). A PCR fragment of the PEDV ORF1 region was amplified using primers F-V/R-V(Table 1) from PEDV classical attenuated vaccine strain CV777 cDNA and named PEDV-V/qRT-PCR. Another PCR fragment of the PEDV ORF1 region was amplified using primers F-W/R-W (Table 1) from PEDV DX cDNA and named PEDV-W/qRT-PCR. The PCR reaction was performed with PrimeSTAR ${ }^{\circledR}$ Max DNA Polymerase kit (Takara, Dalian, China) and the reaction system as follows: $25 \mu \mathrm{L}$ PrimeSTAR ${ }^{\circledR}$ Max Premix $(2 \mathrm{X}), 1.5 \mu \mathrm{L}$ each primer $(50 \mu \mathrm{M}), 2 \mu \mathrm{l} \mathrm{cDNA}$, and $\mathrm{ddH}_{2} \mathrm{O}$ to a total volume of $50 \mu \mathrm{L}$ in each PCR tube, and cycled as follows: 35 cycles of $98^{\circ} \mathrm{C}$ for $10 \mathrm{~s}, 55^{\circ} \mathrm{C}$ for $15 \mathrm{~s}$, and $72^{\circ} \mathrm{C}$ for $10 \mathrm{~s}$. Both recombinant plasmid DNA were constructed by cloning of two PCR fragments into the pET-30a vector (Genecreate, Nanjing, China) and were sequenced by TsingKe (Xian, China), respectively. Both recombinant plasmids were linearized with Ndel (Takara, Dalian, China), purified using the TaKaRa MiniBEST DNA Fragment Purification Kit Version 4.0 (Takara, Dalian, China), and both PEDV RNAs were transcribed in vitro using the RiboMAX Large Scale RNA Production System-T7 (Promega, Madison, WI, USA). The length and integrity of transcribed both standard PEDV RNAs in vitro were verified by Agarose gel electrophoresis. The concentration of both RNA standards were measured using a ND-2000c spectrophotometer (Thermo Fisher Scientific, Waltham, USA) and the RNA copy number was calculated as follows: $\left(6.02 \times 10^{23}\right.$ molecules/mole $) \times($ RNA concentration $) /(340 \times$ number of bases $)$.

\section{The one-step real-time RT-PCR assay}

Real-time RT-PCR assay was carried out using a Bio-Rad CFX Manager (Bio-Rad, USA) instrument. The primers and probes were designed to amplify the PEDV gene ORF1 region in this study or the PEDV S1 domain[12]. The reactions were carried out using the One Step PrimeScript ${ }^{\circledR}$ RT-PCR Kit (Perfect Real Time) (Takara, Dalian, China) with reaction system as follows: $2 \times$ One Step RT-PCR Buffer $12.5 \mu \mathrm{L}$,

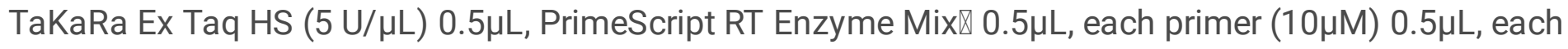
probe $(10 \mu \mathrm{M}) 1 \mu \mathrm{L}, 1 \mu \mathrm{l}$ of viral RNA/DNA or $4 \mu \mathrm{L}$ sample RNA, and $\mathrm{ddH}_{2} \mathrm{O}$ to a total volume of $25 \mu \mathrm{L}$ in each PCR tube, and cycled as follows: $42^{\circ} \mathrm{C}$ for $5 \mathrm{~min}, 95^{\circ} \mathrm{C}$ for $10 \mathrm{~s}$, then 40 cycles of $95^{\circ} \mathrm{C}$ for $5 \mathrm{~s}$ and $60{ }^{\circ} \mathrm{C}$ for $31 \mathrm{~s} \rrbracket$

\section{The one-step RT-PCR assay}

Eight pairs of primers(Table S2) were used for RT-PCR with the PrimeScript "' One Step RT-PCR Kit Ver. 2.0 (Takara, Dalian, China) to detect of major diarrhoeal viruses( PEDV, TGEV, PKV, PPV, PDCoV, and PCV-2) in 117 samples of suspected PEDV infection. This RT-PCR reaction system as follows: PrimeScript one Step Enzyme Mix $2 \mu \mathrm{L}$, $2 \times$ one Step Buffer $25 \mu \mathrm{L}$, each primer 10pmol, $1 \mu \mathrm{l}$ of viral RNA/DNA or $4 \mu \mathrm{L}$ sample RNA/DNA, and $\mathrm{ddH}_{2} \mathrm{O}$ to a total volume of $50 \mu \mathrm{L}$ in each PCR tube, and cycled as follows: $50^{\circ} \mathrm{Cfor} 45 \mathrm{~min}$, 
$94^{\circ} \mathrm{Cfor} 2 \mathrm{~min}$, followed by 35 cycles of $95^{\circ} \mathrm{C}$ for $5 \mathrm{~s}, 55^{\circ} \mathrm{C}$ for $30 \mathrm{~s}$, and $72^{\circ} \mathrm{C}$ for $60 \mathrm{~s}$, with a final extension at $72^{\circ} \mathrm{C}$ for $10 \mathrm{~min}$.

\section{Specificity and sensitivity analysis of PEDV one-step real-time RT-PCR assay}

All viral RNA and DNA samples were quantitated using a ND-2000c spectrophotometer (Thermo Fisher Scientific, Waltham, USA) in our laboratory. Ten nanograms of RNA or DNA extracted from PEDV classical attenuated vaccine strain CV777, Vero-cell-adapted isolate JS2008, PEDV DX, TGEV, PKV, PPV, PDCoV, and PCV-2 in three replicates was used as template for specificity analysis of PEDV one-step real-time RTPCR assay. PEDV one-step real-time RT-PCR assay was performed using a Bio-Rad CFX Manager instrument (Bio-Rad, USA).

For sensitivity analysis of PEDV one-step real-time RT-PCR assay, ten-fold serial dilutions of both RNA standards were used as template (range: $3.0 \times 10^{10}-3.0 \times 10^{1}$ copies). One microliter of each PEDV RNA standard serial dilution (range: $3.0 \times 10^{1}-3.0 \times 10^{10}$ copies) was applied to evaluate the dynamic detection range of one-step real-time RT-PCR assay. Each experiment was repeated three times and regression analysis was performed using Bio-Rad CFX Manager (Bio-Rad, USA) to determine detection limits.

\section{Analysis of clinical samples using PEDV real-time RT-PCR and RT-PCR assay}

A total of $117 \mathrm{fecal}$ samples were collected from seven pig farms with the background of immunizing with CV777-based monovalent or bivalent attenuated vaccines, six of which from six piglets with oral attenuated vaccine CV777 and showed no clinical symptoms of diarrhea, and the remaining $111 \mathrm{fecal}$ samples were obtained from 111 piglets with clinical status of diarrhea. All fecal samples were used to evaluate the reliability of the established PEDV one-step real-time RT-PCR assay. Another real-time RT-PCR and RT-PCR assays were compared, and all results were shown in Table 3. All positive PCR products were sequenced by TsingKe (XiAn, China).

\section{Results}

\section{Specificity, sensitivity, and repeatability analysis of PEDV one-step real time RT-PCR assay}

For the specificity of PEDV one-step real time RT-PCR assay, only PEDV wild-type strain DX showed a fluorescent signal using the primers F3365/R3445 and probe P3390-W (Fig.2.A). only PEDV classical attenuated vaccine strain CV777 or Vero-cell-adapted isolate JS2008 showed fluorescent signals using the primers F3365/R3445 and probe P3388-V(Fig.2.B). However, TGEV, PKV, PPV, PDCoV, and PCV-2 showed no fluorescent signals in this assay, indicating good specificity (Fig.2). The primers F3365/R3445, probes P3390-W, and P3388-V could be combined in a tube for simultaneously detecting PEDV attenuated vaccine strain CV777 and PEDV wild-type strain DX, without cross-reactivity for each other (Fig.3). 
The detection limits for PEDV wild-type strains and classical attenuated vaccine strains were $3.0 \times 10^{3}$ copies/reation (Fig.4) and $3.0 \times 10^{2}$ copies/reation, respectively (Fig.5). Using 10-fold serial dilutions of RNA standard as template, each experiment was repeated three times, to establish standard curves for PEDV wild-type strains (Fig.4.B) and classical attenuated vaccine strains (Fig.5.B). As shown in Table 2, the detection limit of the PEDV wild-type strains were $3.0 \times 10^{3}$ RNA copies/reaction for PEDV wild-type strain DX standard RNA, and the detection limit of the PEDV classical attenuated vaccine strains were $3.0 \times 10^{2}$ RNA copies/reaction for PEDV classical attenuated vaccine strain CV777 standard RNA. The epeatability of this established assay was evaluated using two ORF1 region standard RNAs of PEDV classical attenuated vaccine strain CV777 and wild-type strain DX, respectively, with coefficients of variation 0.50-6.8 (Table 2).

Table 2.The sensitivity and repeatability of established PEDV real-time RT-PCR assay

\begin{tabular}{|c|c|c|c|c|c|c|c|}
\hline $\begin{array}{l}\text { Standard } \\
\text { RNA }\end{array}$ & $\begin{array}{l}\text { Copy } \\
\text { number }\end{array}$ & $\begin{array}{l}\mathrm{Cq} \\
(\text { mean } \pm \text { S.D.) }\end{array}$ & CV\% & $\begin{array}{l}\text { Standard } \\
\text { RNA }\end{array}$ & $\begin{array}{l}\text { Copy } \\
\text { number }\end{array}$ & $\begin{array}{l}\mathrm{Cq} \\
\text { (mean } \pm \text { S.D.) }\end{array}$ & CV\% \\
\hline \multirow{6}{*}{$\begin{array}{l}\text { ORF1 of } \\
\text { PEDV }\end{array}$} & $3.0 \times 10^{8}$ & $13.30 \pm 0.52$ & 3.90 & \multirow{9}{*}{$\begin{array}{l}\text { ORF1 of } \\
\text { PEDV } \\
\text { CV777 }^{\text {b }}\end{array}$} & $3.0 \times 10^{8}$ & $12.49 \pm 0.85$ & 6.8 \\
\hline & $3.0 \times 10^{7}$ & $16.17 \pm 0.59$ & 3.65 & & $3.0 \times 10^{7}$ & $16.41 \pm 0.09$ & 0.55 \\
\hline & $3.0 \times 10^{6}$ & $18.37 \pm 0.26$ & 1.42 & & $3.0 \times 10^{6}$ & $19.19 \pm 0.33$ & 1.72 \\
\hline & $3.0 \times 10^{5}$ & $21.67 \pm 0.33$ & 1.52 & & $3.0 \times 10^{5}$ & $22.76 \pm 0.24$ & 1.05 \\
\hline & $3.0 \times 10^{4}$ & $24.72 \pm 0.38$ & 1.54 & & $3.0 \times 10^{4}$ & $25.42 \pm 0.27$ & 1.06 \\
\hline & $3.0 \times 10^{3}$ & $26.15 \pm 0.26$ & 0.94 & & $3.0 \times 10^{3}$ & $29.15 \pm 0.29$ & 0.99 \\
\hline \multirow{4}{*}{$\mathrm{DX}^{\mathrm{a}}$} & $3.0 \times 10^{2}$ & None & None & & $3.0 \times 10^{2}$ & $32.12 \pm 0.16$ & 0.50 \\
\hline & $3.0 \times 10^{1}$ & None & None & & $3.0 \times 10^{1}$ & None & None \\
\hline & $3.0 \times 10^{0}$ & None & None & & $3.0 \times 10^{0}$ & None & None \\
\hline & NCT & None & None & & NCT & None & None \\
\hline
\end{tabular}

a, Related to the ORF1 region of PEDV wild-type strain DX, the Standard RNA as template was used for detection of PEDV wild-type strains.

b, Related to the ORF1 region of PEDV classical attenuated vaccine strain CV777, the Standard RNA as template was used detection of PEDV classical attenuated vaccine strains

Evaluation of real time RT-PCR and RT-PCR assays using clinical samples 
117 fecal samples from 117 pigs with suspected PEDV infection were subjected to PEDV one-step realtime RT-PCR (a), real-time RT-PCR (b), and RT-PCR assays (c). PEDV wild-type strains were detected in 83.76, 83.76, and $79.80 \%$ of all samples, respectively, and PEDV classical attenuated vaccine strains were detected in $6.84,6.84$ and $5.98 \%$ of all samples, respectively (Table 3 ). As shown in Table 3 , of the 17 samples that tested negative in the RT-PCR assay(c), 11 samples were PEDV-negative and the remaining 6 samples were PEDV-positive (five wild-type strains and one classical attenuated vaccine strain) as detected by the one-step real-time RT-PCR (a) and real-time RT-PCR assays (b). As shown in Fig.6, through the analysis of the established one-step real-time RT-PCR assay, the viral load of 106 PEDVpositive samples was concentrated between $10^{3.265}-10^{6}$ copies/reation. Sequencing results for all PEDVpositive samples indicated that the PEDV one-step real time RT-PCR assay (a) had high specificity and sensitivity. In addition, TGEV and PKV were detected in the 11 PEDV-negative samples and 8 PEDVpositive samples (detailed data no shown), indicating that the co-infections of multiple diarrhea viruses have occurred in some pig farms.

Table 3. Results for 117 samples in the three assays.

\begin{tabular}{|llllllll|}
\hline location & Samples & One-step qRT-PCR(a) & qRT-PCR(b) & \multicolumn{2}{l|}{ RT-PCR(c) } \\
\cline { 5 - 8 } in & number & & & & & & \\
Gansu Province & & wild & vaccine & wild & vaccine & wild & vaccine \\
& & strain & strain & strain & strain & strain & strain \\
\hline DingXI & 25 & 24 & 0 & 24 & 0 & 23 & 0 \\
\hline JiayuGuan & 19 & 15 & 2 & 15 & 2 & 14 & 2 \\
\hline LinXia & 23 & 17 & 3 & 17 & 3 & 16 & 2 \\
\hline TianShui & 13 & 9 & 2 & 9 & 2 & 9 & 2 \\
\hline BaiYin & 20 & 19 & 0 & 19 & 0 & 19 & 0 \\
\hline LanZhou & 17 & 14 & 1 & 14 & 1 & 13 & 1 \\
\hline total & 117 & 98 & 8 & 98 & 8 & 93 & 7 \\
\hline The positive rate & & $83.76 \%$ & $6.84 \%$ & $83.76 \%$ & $6.84 \%$ & $79.80 \%$ & $5.98 \%$ \\
\hline
\end{tabular}

1. Method established in this study

2. Method from Su et al., 2018[12]

3. Method from He et al., 2019[13]

\section{Discussion}


Despite the current vaccination policy in China, multiple types of PEDV strains still appeared in pig farms, especially the variant strains that began in southern China in 2010, causing huge losses to the pig industry $[6,13]$. A recent phylogenetic tree study of Chinese PEDV strains and some PEDV representative strains of other countries revealed that the full-length genomic sequences can be divided into two independent subgroups, namely GI (classical strains, GI-a and GI-b) and GII (variant strains, GII-a, and GIIb) $[17,32]$. The PEDV strains of the GI-b subgroup contained Vero-cell-adapted vaccine strains (PEDV attenuated vaccine KC189944, attenuated CV777 and DR13) and five Vero-cell-adapted isolates (JS2008, SDM, SQ2014, SC1402, HLJBY)[15, 17, 32-36]. By alignment analysis of ORF1 region sequence in the 46 PEDV strains released by GenBank (Fig.1 and Table S1), we found that three classical attenuated vaccine strains (KC189944, attenuated strain CV777 and DR13, GI-b) and Vero-cell-adapted isolates (JS2008, SC1402, SQ2014, HLJBY and SDM, GI-b) have a novel 24-nucleotide deletion in the N-terminal domain of ORF1 region nsp3 compared with 38 wild-type PEDV strains (GI-a and GII). PEDV Vero-celladapted vaccine strains belonging to GI-b subgroup not only have nucleotides variation in the ORF3[16], but there are also 24-nt deletions in the ORF1 region (Fig.1 and Table S1). Based on these discoveries, a one-step real-time RT-PCR assay was developed to distinguish PEDV classical attenuated vaccine strains and wild-type strains. The 24-nucleotide-deletion pattern in the ORF1 region of PEDV Vero cell-adapted strains could be the marker of adaptation to Vero cell culture, and it is a valuable tool for monitoring of the persistence of classical attenuated vaccine strains and epidemiologic research of PEDV infection in swine herds as well as stability and safety analysis of classical attenuated vaccines. However, there are some situations that need our attention. For example, since the ORF1 sequence of some candidate vaccines has not been reported, especially the vaccine candidates from highly virulent strains (genotype 2a) emerged after 2010, we are not sure whether the ORF1 of these vaccine candidates derived from nonclassical attenuated vaccine strains have the same 24 nucleotides deletion pattern. If this pattern exists in all cell-adapted strains during continuous passages in Vero cells, it can be used as a genotyping marker just like nucleotide deletions of Spike or ORF3 genes in the Vero-cell-adapted strains[37, 38]. Moreover, multiple PEDV strains can co-exist in some co-infection events, and it is possible that the wildtype virus repairs the $24 \mathrm{nt}$ deleted region of the classical attenuated vaccine strain. If the 24 nucleotides deletion pattern of the attenuated vaccine strains is repaired, the method we established will not apply. Nevertheless, Commercial vaccines based on classical strains are extensively applied in Chinese pig farms and play a very important role in controlling PEDV infections, while vaccines derived from nonclassical attenuated vaccine candidates are being developed and not yet commercially available. Therefore, our method is safe, accurate, and reliable, and can be applied to the identification of classical attenuated vaccine strains and the safety evaluation of attenuated vaccines in pig farms

The one-step real-time RT-PCR assay could only detect PEDV, with no cross-reactivity with other enteroviruses. The limits of detection for PEDV wild-type strains and classical attenuated vaccine strains in the one-step real-time RT-PCR assay were $3.0 \times 10^{3}$ copies and $3.0 \times 10^{2}$ copies, respectively. Compared with the other two methods (Table 3), the detection results of $117 \mathrm{fecal}$ samples showed that the our established assay had highly PEDV positive diagnosis agreement with real-time RT-PCR(100\%) and RTPCR assays(95.6\%) (Table 3), respectively, indicating that the established one-step real-time RT-PCR 
assay have high sensitivity, rapidity, and accuracy. The detection results of $117 \mathrm{fecal}$ samples in our established one-step real-time RT-PCR assay, 98 PEDV wild-type strains were detected, including PEDV classical wild strains and variants, 8 PEDV classical attenuated vaccine strains were detected, and TGEV and PKV were detected in the 11 PEDV-negative samples and 8 PEDV-positive samples (data no shown). The sequencing results of 8 positive samples tested as classical attenuated strains in this study showed that they have the same nucleotide deletion positions as the ORF1 and ORF3 fragments in the classical attenuated vaccine CV777. These results showed that PEDV attenuated vaccines have existed in Gansu province, China and the co-infections of multiple diarrhea viruses have occurred in some pig farms. Therefore, it is necessary to take measures to dynamically monitor the co-infection of other enteric pathogens with PEDV in pig farms. Besides, We did not successfully isolate live virus using Vero-E6 cells in the positive samples tested as PEDV classical attenuated vaccine strains. This may be due to low live virus content or only nucleic acid fragments in the sample.

\section{Conclusion}

This one-step real-time RT-PCR assay was developed to distinguish between classical attenuated vaccine strains that were artificially inoculated and wild-type strains during epidemiological surveillance, and to provide technical support for collecting more accurate epidemiological data of PEDV infection. Since, various PEDV strains may coexist in the same environment, even the same pig, which may cause the vaccine strain to revert to virulence by genetic recombination with other types of PEDV strains. Thus, monitoring the spread of attenuated vaccine strains and changes in virus titer could provide an important reference for evaluating immune protection effect of attenuated vaccine strains and the prevention and control of PEDV. Addtionally, our method only use a pair of primers and two probes that placed in one reaction tube to detect different types of PEDV strains, without cross-reactivity between each other. When distinguishing different types of PEDV strains in a sample, a tube of amplification enzyme, a pairs of primers, and a tube of PCR reaction tube can be save, and this also reduced the cost of detecting sample in the commercial application. Moreover, this method allows quantitative calculation of viral load, and has practical value for epidemiological investigations of wild-type strains and classical attenuated vaccine strains. Simultaneously, combined with other methods for detecting PEDV, it can be effective for the genotyping and prevalence of various strains of PEDV. Finally, it also represents an alternative detection tool for preliminary identification of clinical samples.

\section{Abbreviations}

PEDV: Porcine epidemic diarrhea virus; PED: Porcine epidemic diarrhea; PCV-2: Porcine circovirus type 2; PDCoV: Porcine deltacoronavirus; PKV: Porcine kobuvirus; PPV: Porcine parvovirus; TGEV: Transmissible gastroenteritis virus;N: Nucleocapsid; S: Spike; ORF3: Open reading frame 3; E: Envelope;M: Membrane; nsp3: non-structural protein; S-INDEL: Insertion and deletion in the S gene; CV: Coefficients of variation; SD: Standard deviation; RT-PCR: Reverse transcription-polymerase chain re-action; PCR: Polymerase chain reaction 


\section{Declarations}

\section{Ethics approval and consent to participate}

All animals were handled in strict accordance with good animal practice according to the Animal Ethics Procedures and Guidelines of the People's Republic of China, and the study was approved in writing by the Animal Ethics Committee of Lanzhou Veterinary Research Institute, Chinese

Academy of Agricultural Sciences (No. LVRIAEC 2016-003). The Animal Ethics Committee of Lanzhou Veterinary Research Institute, Chinese Academy of Agricultural Sciences approved the collection of fecal samples after obtaining verbal consent from the swine farm owners

\section{Consent for publication}

Not applicable.

\section{Author's contribution}

Xi Lan collected the samples in Gansu Province and designed the study; Zhilin Wang performed the research; Zhilin Wang, Xuerui Li, Youjun Shang, Zhen Dong, Wanning Wang, and Yongsheng Liu analyzed data; Zhilin Wang drafted the manuscript. All authors read and agreed the final manuscript

\section{Conflict of interest}

The authors declare that they have no competing interests. The work is an original paper and is not under consideration in other journals.

\section{Funding}

This work was supported by National Key Research and Development Program (2016YFD0500703, 2017YFD0500903)

\section{Availability of data and material}

The data of this study are available from the corresponding author on reasonable request.

\section{Additional file}

All data generated or analysed during this study are included in this published article (and its additional file Table S1 and Table S2)

Table S1. The name, accession number, and search website of the PEDV strain in the study.

Table S2. Additional primers and probes of detecting PEDV, TGEV, PKV, PPV, PDCoV, and PCV-2 in the study. 
Acknowledgements

Not applicable.

\section{References}

1. Pensaert MB, de Bouck P: A new coronavirus-like particle associated with diarrhea in swine.Arch Virol 1978, 58:243-247.

2. Wood EN: An apparently new syndrome of porcine epidemic diarrhoea. Vet Rec 1977, 100:243-244.

3. Duarte M, Tobler K, Bridgen A, Rasschaert D, Ackermann M, Laude H: Sequence analysis of the porcine epidemic diarrhea virus genome between the nucleocapsid and spike protein genes reveals a polymorphic ORF.Virology 1994, 198:466-476.

4. Chen J, Wang C, Shi H, Qiu H, Liu S, Chen X, Zhang Z, Feng L: Molecular epidemiology of porcine epidemic diarrhea virus in China.Arch Virol 2010, 155:1471-1476.

5. Hanke D, Jenckel M, Petrov A, Ritzmann M, Stadler J, Akimkin V, Blome S, Pohlmann A, Schirrmeier H, Beer $M$, Hoper D: Comparison of porcine epidemic diarrhea viruses from Germany and the United States, 2014.Emerg Infect Dis 2015, 21:493-496.

6. Li W, Li H, Liu Y, Pan Y, Deng F, Song Y, Tang X, He Q: New variants of porcine epidemic diarrhea virus, China, 2011.Emerg Infect Dis 2012, 18:1350-1353.

7. Song D, Park B: Porcine epidemic diarrhoea virus: a comprehensive review of molecular epidemiology, diagnosis, and vaccines. Virus Genes 2012, 44:167-175.

8. Sun RQ, Cai RJ, Chen YQ, Liang PS, Chen DK, Song CX: Outbreak of porcine epidemic diarrhea in suckling piglets, China.Emerg Infect Dis 2012, 18:161-163.

9. Gerdts V, Zakhartchouk A: Vaccines for porcine epidemic diarrhea virus and other swine coronaviruses. Vet Microbio/ 2017, 206:45-51.

10. Shi D, Lv M, Chen J, Shi H, Zhang S, Zhang X, Feng L: Molecular characterizations of subcellular localization signals in the nucleocapsid protein of porcine epidemic diarrhea virus. Viruses 2014, 6:1253-1273.

11. Sun D, Wang $X$, Wei S, Chen J, Feng L: Epidemiology and vaccine of porcine epidemic diarrhea virus in China: a mini-review.J Vet Med Sci 2016, 78:355-363.

12. Su Y, Liu Y, Chen Y, Xing G, Hao H, Wei Q, Liang Y, Xie W, Li D, Huang H, et al: A novel duplex TaqMan probe-based real-time RT-qPCR for detecting and differentiating classical and variant porcine epidemic diarrhea viruses.Mol Cell Probes 2018, 37:6-11.

13. He D, Chen F, Ku X, Yu X, Li B, Li Z, Sun Q, Fan S, He Q: Establishment and application of a multiplex RT-PCR to differentiate wild-type and vaccine strains of porcine epidemic diarrhea virus.J Virol Methods 2019, 272:113684.

14. Zhu Y, Wang GH, Cui YD, Cui SJ: Establishment of a nanoparticle-assisted RT-PCR assay to distinguish field strains and attenuated strains of porcine epidemic diarrhea virus.Arch Virol 2016, 
161:2543-2547.

15. Zhang H, Xia M, Ju D, Wu B, Ning C, Song N, Feng T, Chen F, Wang X, Wu Y, et al: Isolation, molecular characterization and an artificial infection model for a variant porcine epidemic diarrhea virus strain from Jiangsu Province, China.Arch Virol 2017, 162:3611-3618.

16. Sun M, Ma J, Wang Y, Wang M, Song W, Zhang W, Lu C, Yao H: Genomic and epidemiological characteristics provide new insights into the phylogeographical and spatiotemporal spread of porcine epidemic diarrhea virus in Asia.J Clin Microbio/ 2015, 53:1484-1492.

17. Wang D, Fang L, Xiao S: Porcine epidemic diarrhea in China.Virus Res 2016, 226:7-13.

18. Bridgen A, Kocherhans $\mathrm{R}$, Tobler $\mathrm{K}$, Carvajal A, Ackermann M: Further analysis of the genome of porcine epidemic diarrhoea virus.Adv Exp Med Bio/ 1998, 440:781-786.

19. Kocherhans R, Bridgen A, Ackermann M, Tobler K: Completion of the porcine epidemic diarrhoea coronavirus (PEDV) genome sequence. Virus Genes 2001, 23:137-144.

20. Brian DA, Baric RS: Coronavirus genome structure and replication.Curr Top Microbiol Immuno/ 2005, 287:1-30.

21. Hagemeijer MC, Monastyrska I, Griffith J, van der Sluijs P, Voortman J, van Bergen en Henegouwen PM, Vonk AM, Rottier PJ, Reggiori F, de Haan CA: Membrane rearrangements mediated by coronavirus nonstructural proteins 3 and 4. Virology 2014, 458-459:125-135.

22. Baez-Santos YM, St John SE, Mesecar AD: The SARS-coronavirus papain-like protease: structure, function and inhibition by designed antiviral compounds. Antiviral Res 2015, 115:21-38.

23. Sung MH, Deng MC, Chung YH, Huang YL, Chang CY, Lan YC, Chou HL, Chao DY: Evolutionary characterization of the emerging porcine epidemic diarrhea virus worldwide and 2014 epidemic in Taiwan.Infect Genet Evol 2015, 36:108-115.

24. Ishikawa K, Sekiguchi H, Ogino T, Suzuki S: Direct and rapid detection of porcine epidemic diarrhea virus by RT-PCR.J Virol Methods 1997, 69:191-195.

25. Yu X, Shi L, Lv X, Yao W, Cao M, Yu H, Wang X, Zheng S: Development of a real-time reverse transcription loop-mediated isothermal amplification method for the rapid detection of porcine epidemic diarrhea virus. Virol $J$ 2015, 12:76.

26. Miller LC, Crawford KK, Lager KM, Kellner SG, Brockmeier SL: Evaluation of two real-time polymerase chain reaction assays for Porcine epidemic diarrhea virus (PEDV) to assess PEDV transmission in growing pigs.J Vet Diagn Invest 2016, 28:20-29.

27. Liu J, Li LM, Han JQ, Sun TR, Zhao X, Xu RT, Song QY: A TaqMan probe-based real-time PCR to differentiate porcine epidemic diarrhea virus virulent strains from attenuated vaccine strains. $\mathrm{Mol}$ Cell Probes 2019, 45:37-42.

28. Lee C, Park CK, Lyoo YS, Lee du S: Genetic differentiation of the nucleocapsid protein of Korean isolates of porcine epidemic diarrhoea virus by RT-PCR based restriction fragment length polymorphism analysis. Vet $J$ 2008, 178:138-140. 
29. Jung K, Chae C: RT-PCR-based dot blot hybridization for the detection and differentiation between porcine epidemic diarrhea virus and transmissible gastroenteritis virus in fecal samples using a nonradioactive digoxigenin cDNA probe.J Virol Methods 2005, 123:141-146.

30. Zhao PD, Bai J, Jiang P, Tang TS, Li Y, Tan C, Shi X: Development of a multiplex TaqMan probe-based real-time PCR for discrimination of variant and classical porcine epidemic diarrhea virus.J Virol Methods 2014, 206:150-155.

31. Wang Z, Li X, Shang Y, Wu J, Dong Z, Cao X, Liu Y, Lan X: Rapid differentiation of PEDV wild-type strains and classical attenuated vaccine strains by fluorescent probe-based reverse transcription recombinase polymerase amplification assay.BMC Vet Res 2020, 16:208.

32. Guo J, Fang L, Ye X, Chen J, Xu S, Zhu X, Miao Y, Wang D, Xiao S: Evolutionary and genotypic analyses of global porcine epidemic diarrhea virus strains. Transbound Emerg Dis 2019, 66:111-118.

33. Zhao M, Sun Z, Zhang Y, Wang G, Wang H, Yang F, Tian F, Jiang S: Complete genome sequence of a Vero cell-adapted isolate of porcine epidemic diarrhea virus in eastern China.J Virol 2012, 86:1385813859.

34. Li B, Liu H, He K, Guo R, Ni Y, Du L, Wen L, Zhang X, Yu Z, Zhou J, et al: Complete genome sequence of a recombinant porcine epidemic diarrhea virus strain from eastern china.Genome Announc 2013, 1:e0010513.

35. Yang W, Li G, Ren Y, Suo S, Ren X: Phylogeny and expression of the nucleocapsid gene of porcine epidemic diarrhoea virus.Acta Vet Hung 2013, 61:257-269.

36. Huan C, Pan H, Fu S, Xu W, Gao Q, Wang X, Gao S, Chen C, Liu X: Characterization and evolution of the coronavirus porcine epidemic diarrhoea virus HLJBY isolated in China.Transbound Emerg Dis 2020, 67:65-79.

37. Song DS, Yang JS, Oh JS, Han JH, Park BK: Differentiation of a Vero cell adapted porcine epidemic diarrhea virus from Korean field strains by restriction fragment length polymorphism analysis of ORF 3.Vaccine 2003, 21:1833-1842.

38. Oka T, Saif LJ, Marthaler D, Esseili MA, Meulia T, Lin CM, Vlasova AN, Jung K, Zhang Y, Wang Q: Cell culture isolation and sequence analysis of genetically diverse US porcine epidemic diarrhea virus strains including a novel strain with a large deletion in the spike gene. Vet Microbio/ 2014, 173:258269.

\section{Figures}




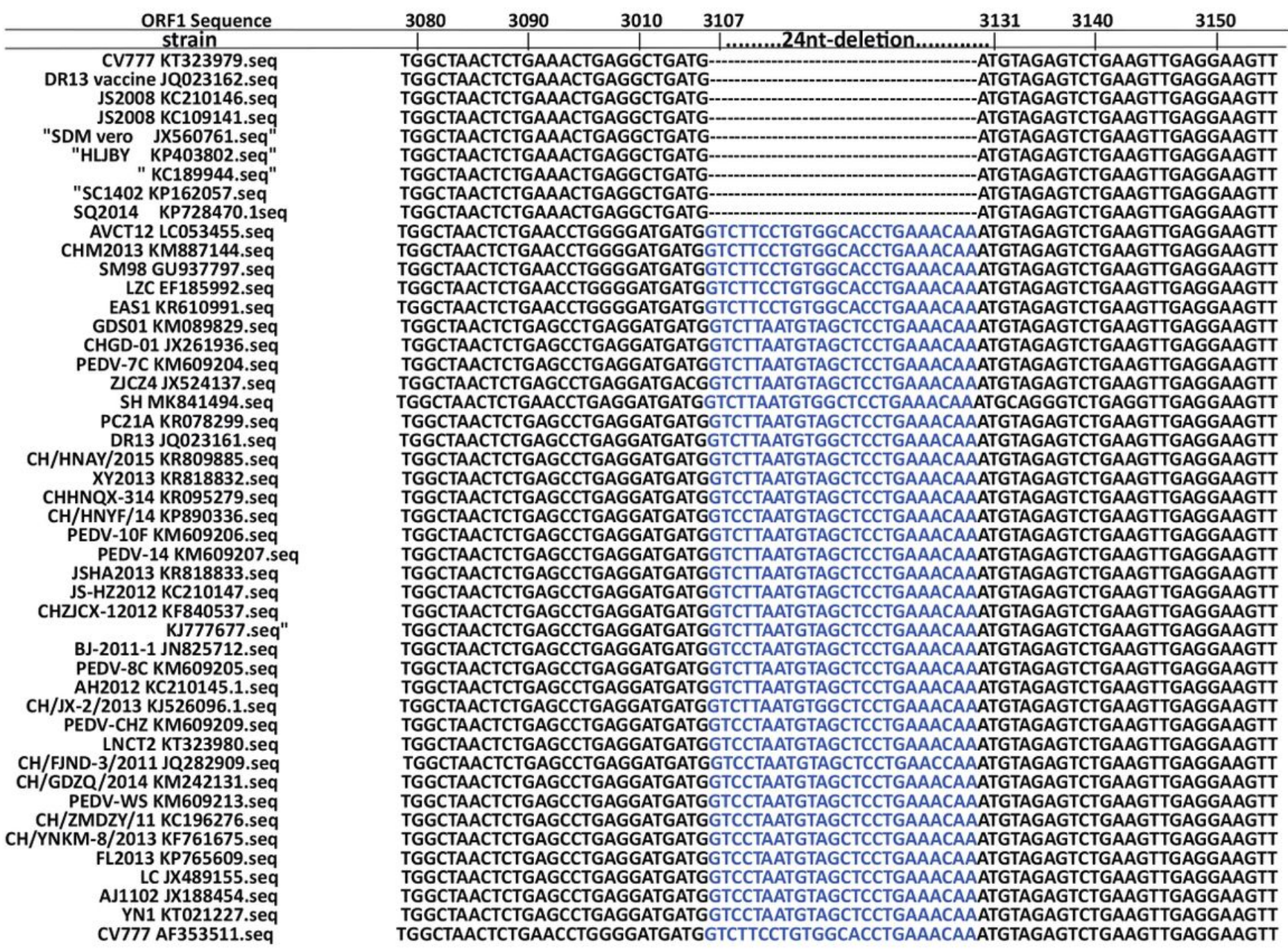

\section{Figure 1}

ORF1 regions alignment results of 38 PEDV wild-types strains and 8 Vero cell-adapted strains whose sequences were available in GenBank, and 24-nucleotide deletions in the ORF1 regions of three PEDV classical attenuated vaccine strains and five Vero-cell-adapted isolates.

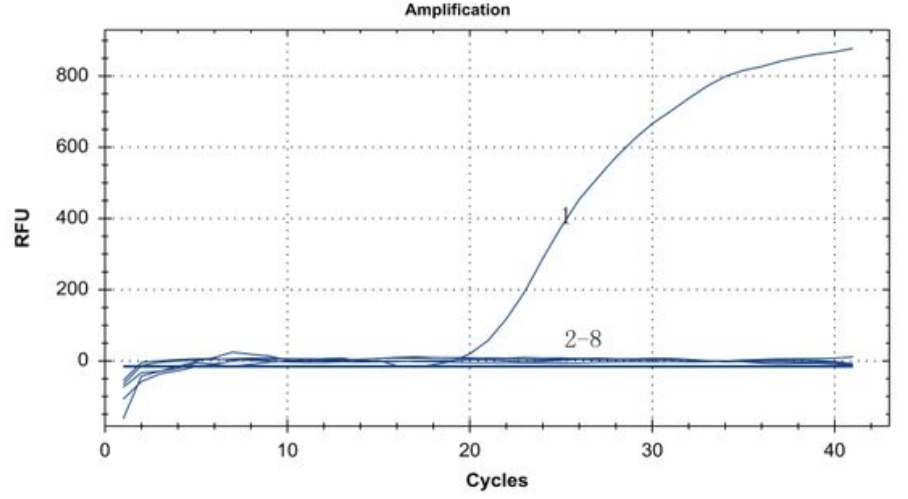

B

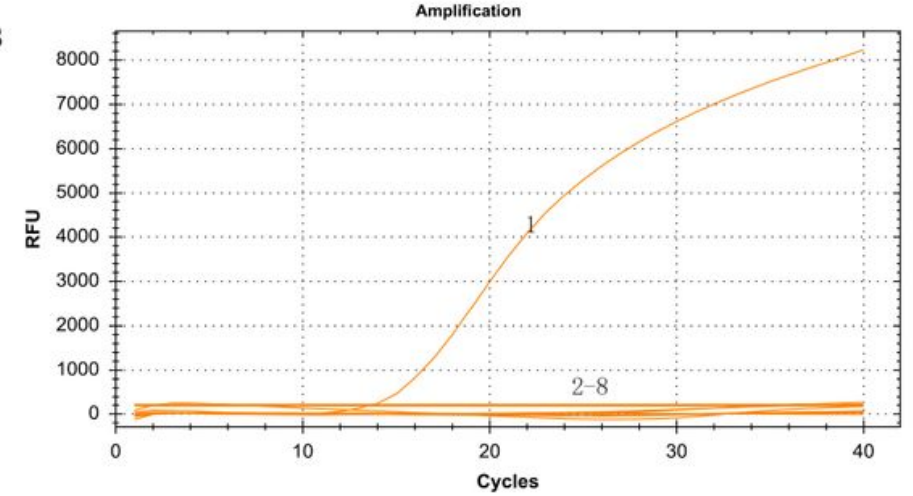

Figure 2 
A. Specificity for PEDV wild-type strain of the one-step real time RT-PCR assay. line 1: PEDV DX; lines 2-8: PEDV classical attenuated vaccine strain CV777 or Vero-cell-adapted isolate JS2008, TGEV, PKV, PPV, PDCoV, and PCV-2 and negative control, respectively. B. Specificity for PEDV classical attenuated vaccine strains of the one-step real time RT-PCR assay. line 1: PEDV classical attenuated vaccine strain CV777 or Vero-cell-adapted isolate JS2008; lines 2-8: PEDV DX, TGEV, PKV, PPV, PDCoV, and PCV-2, and negative control, respectively.

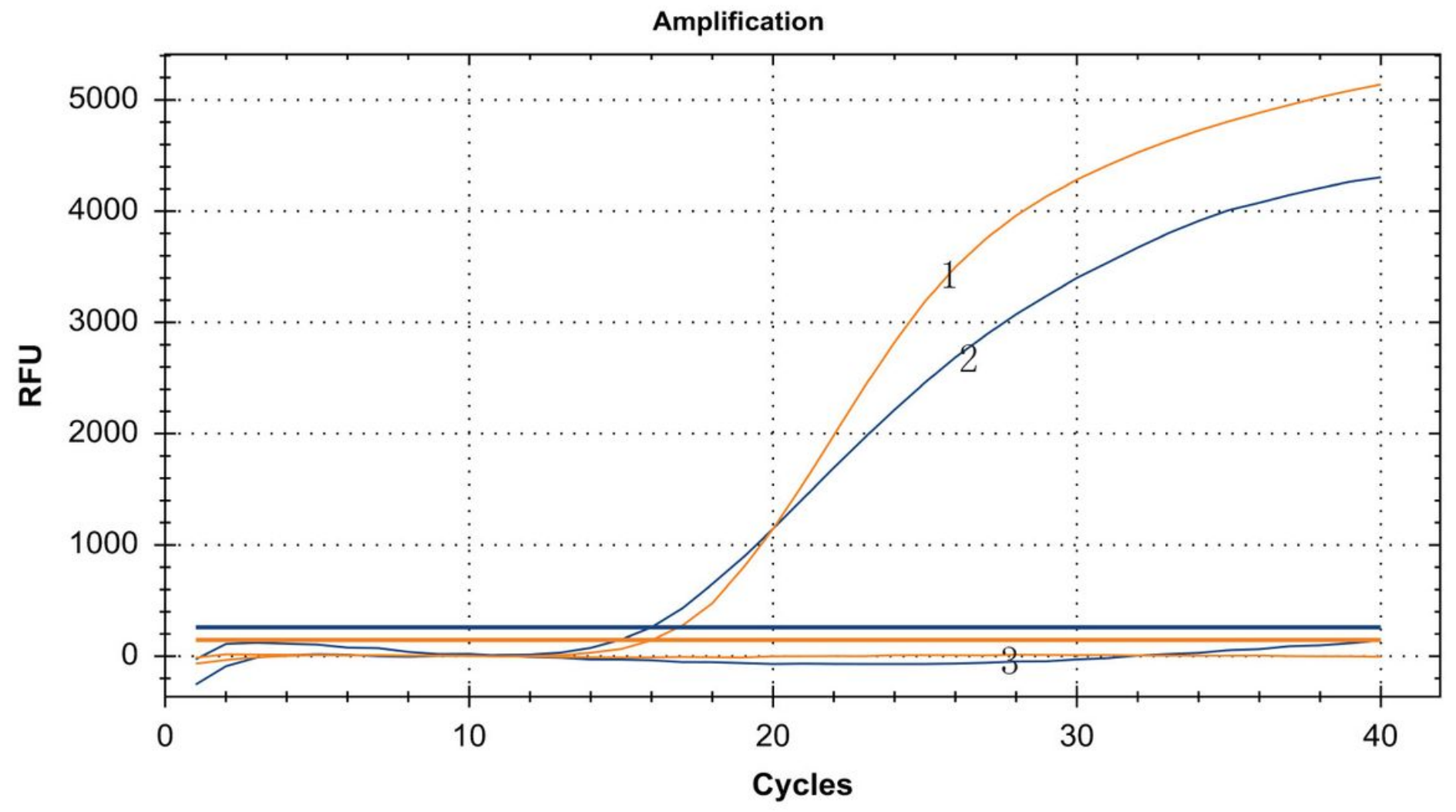

\section{Figure 3}

Specificity of primers and probes in a PCR reaction tube: 1-2: PEDV classical attenuated vaccine strain CV777 RNA,Vero-cell-adapted isolate JS2008, wild-type strain DX RNA, two probes, and a pair of primers were placed in a PCR reaction tube ; 3 : ddH20, two probes, and a pair of primers.

A

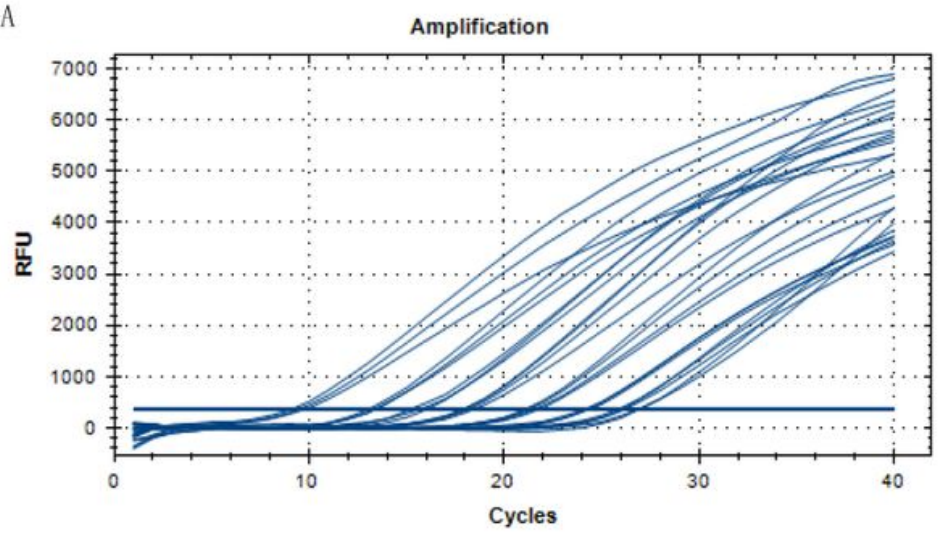

B

Standard Curve

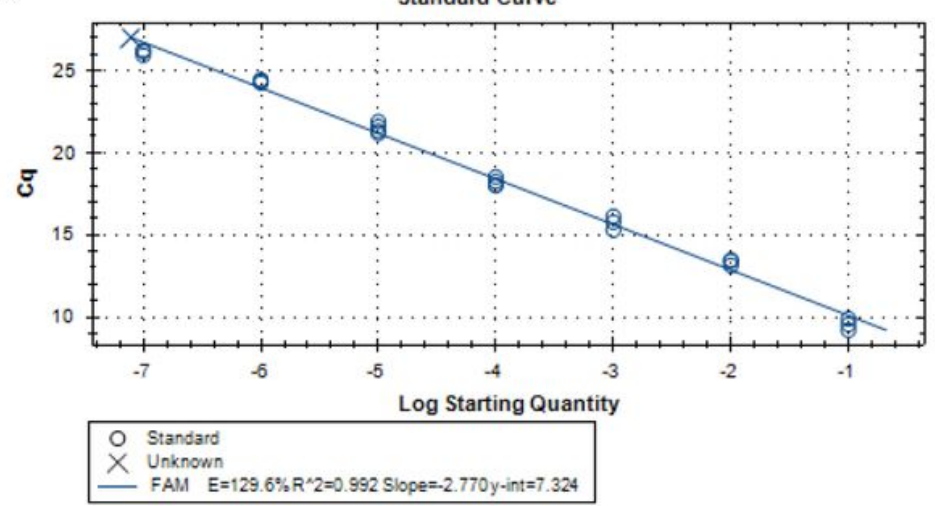

Figure 4 
Sensitivity for PEDV wild-type strains of the one-step real-time RT-PCR. A. Fluorescence development over time using a dilution range of 107-101 copies of the RNA standard (dilution gradient of 10-1-10-7). B. Standard curves of detection of PEDV wild-type strains in the one-step real-time RT-PCR assay. Linear regression of the data provided a formula between RNA copy number (The abscissa represent 10-fold dilution gradient $)$ and $\mathrm{Cq}(\mathrm{Ct})$.

A

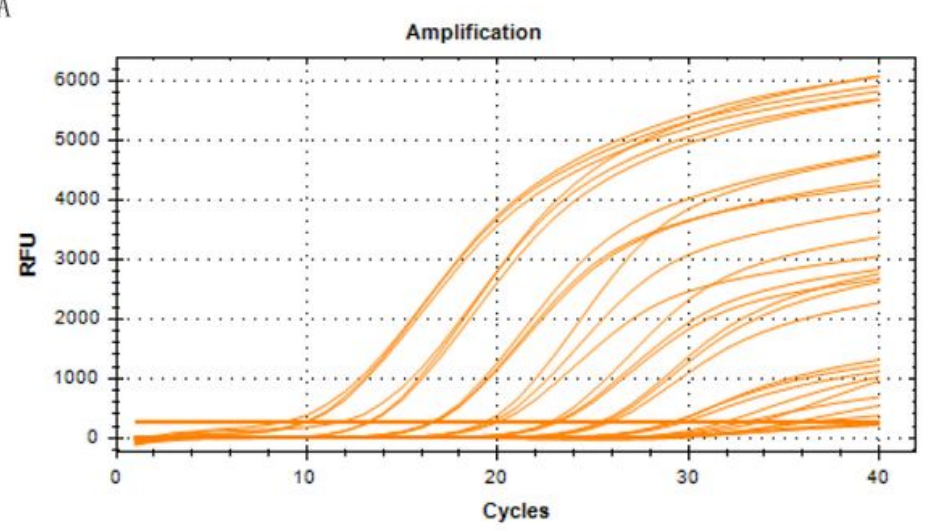

B

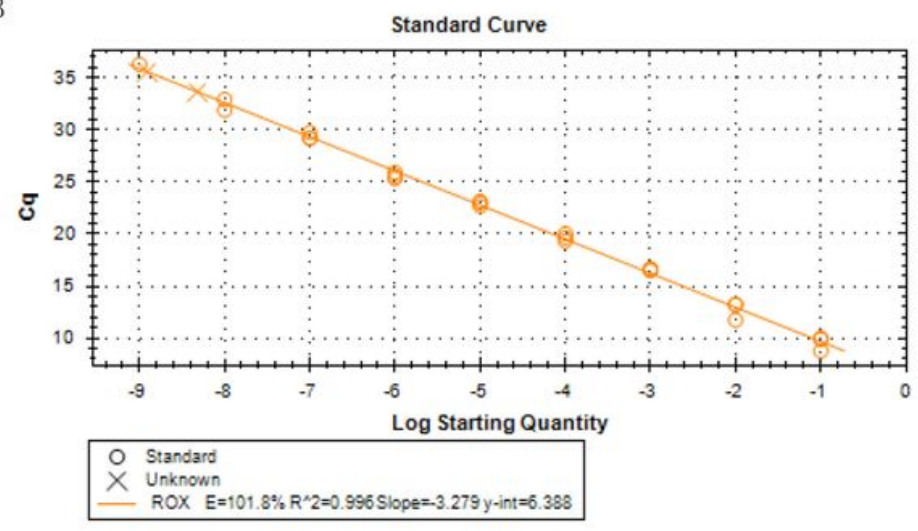

\section{Figure 5}

Sensitivity for PEDV classical attenuated vaccine strains of the one-step real-time RT-PCR. A. Fluorescence development over time using a dilution range of 109-101 copies of the RNA standard (dilution gradient of 10-1-10-9). B. Standard curves of detection of PEDV classical attenuated vaccine strains in the one-step real-time RT-PCR assay. Linear regression of the data provided a formula of between RNA copy number and $\mathrm{Cq}(\mathrm{Ct})$. 


\section{PEDV viral load}

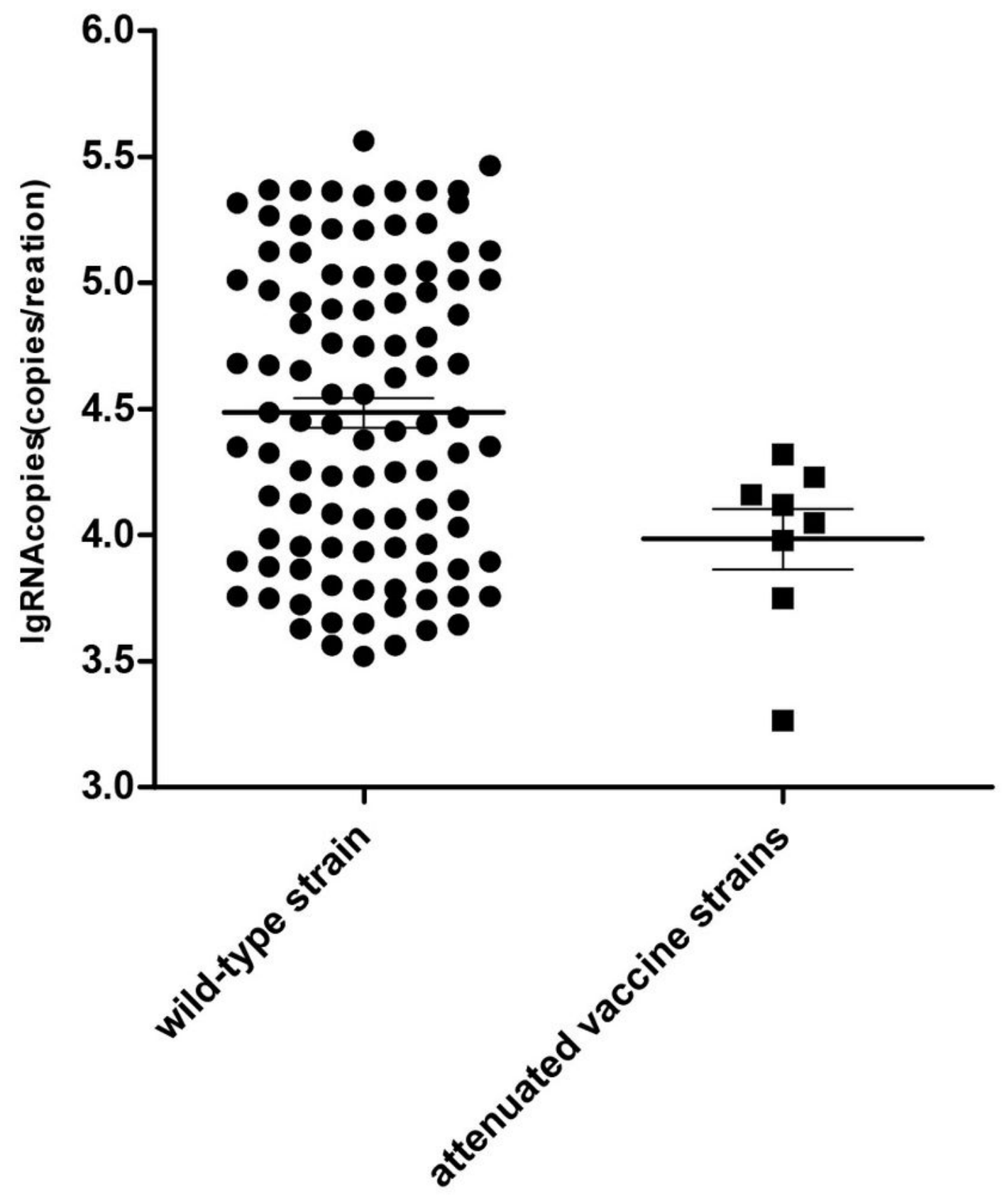

Figure 6

PEDV viral load of each PEDV-positive clinical sample in the established real time RT-PCR assay in this study

Supplementary Files 
This is a list of supplementary files associated with this preprint. Click to download.

- Manuscriptrevisioninstructions.docx

- Tables1.pdf

- Tables2.pdf 